\title{
Thermodynamics of a quark-gluon plasma at finite baryon density.
}

\author{
Z.V Khaidukov ${ }^{+*}$ and Yu.A.Simonov* \\ ${ }^{+}$Moscow Institute of Physics and Technology, Institutskiy pereulok 9, 141700 Dolgoprudny, \\ Moscow Region, Russia \\ *Institute for Theoretical and Experimental Physics,NRC "Kurchatov Institute", B. \\ Cheremushkinskaya 25, Moscow, 117259, Russia
}

September 1, 2021

\begin{abstract}
Properties of the quark-gluon plasma(QGP) in the presence of the baryon chemical potential $\mu_{B}$ are studied using the Field Correlator Method(FCM). The non-perturbative FCM dynamics includes the Polyakov line, computed via colorelectric string tension $\sigma^{E}(T)$ and the quark and gluon Debye masses, defined by the colormagnetic string tension $\sigma^{H}(T)$. The resulting QGP thermodynamics at $\mu_{B} \leq 400 \mathrm{MeV}$ is in a good agreement with the available lattice data,both pressure and the sound velocity do not show any sign of a critical behavior in this region.
\end{abstract}

\section{Introduction}

The main result of heavy ion experiments, performed over the last 15 years at RHIC and then at RHIC and LHC, is the discovery of a new form of matter [1 5 with its properties markedly different from the pre-RHIC era predictions, see [6 15] and references therein. Instead of the commonly assumed picture of a weakly interacting Quark-Gluon Plasma(QGP), one possibly has a strongly coupled liquid subject to the law of the relativistic hydrodynamics [16 18]. The properties of the produced matter drastically change during several stages of evolution: from the stage of formation, hydrodynamization and thermalization towards the hadron gas production. The wealth of the QCD matter phases is reflected in the QCD phase diagram drawn in the $(\mu, T)$ plane. However, the correspondence between the specific $(\mu, T)$ domains of the phase diagram and the space-time dynamics of the fireball should be considered with caution. The reason is that the phase diagram describes the limit of an infinite system in thermodynamic equilibrium.

From the theoretical viewpoint the matter created in heavy ion collisions should be described by the fundamental laws of QCD. For these reasons the dynamics and thermodynamics of QCD at finite temperatures is now in the focus of numerous investigations. At this moment one of the main sources of information is the lattice calculations. The presence of strong interaction in QGP at zero baryon density was demonstrated in numerous studies [19 24]. They show that the ratio of the QGP pressure to the non-interacting case is less than 0.8 and remains almost constant with increasing temperature.

Another striking discovery in this domain was the analysis of the temperature transition, made in the $2+1$ QCD lattice computations, which has shown a smooth crossover in the temperature region $T=140 \div 180 \mathrm{MeV}[25]^{1}$

Despite a dramatic progress the question about the structure of the QCD phase diagram at nonzero baryon density remains open. This happens mostly because lattice methods are strongly restricted to a domain of small chemical potentials ${ }^{2}\left(N_{c}=3\right)$ due to the "sign problem". To circumvent this difficulty

\footnotetext{
${ }^{1}$ This QCD crossover is a new phenomenon, possibly having some analogues in the material sciences and in the ionization and dissociation processes. The question of the existence of a critical point at finite baryon chemical potential is still of intense interest 26 .

${ }^{2}$ We want to point out that another very important source of information of QCD phase diagram is connected with neutron stars physics 27. LIGO and Virgo's discovery of gravitational waves from neutron star 28 opened a new era
} 
in the case of $N_{c}=3$ one can use the Taylor expansion around zero chemical potential [31,32], or use imaginary chemical potential [33]. Another possibility is to decrease the number of colours to $N_{c}=2$, where the sign problem is absent 3437$]$.

From all these facts the need for analytic methods that can help with investigation of QGP thermodynamics and QCD phase diagram becomes obvious. In this paper we will focus on the Field Correlator Method (FCM), which is applicable in QCD at any chemical potential and any temperature [38 43]. In this method the non-perturbative dynamics in confinement and deconfinement regions is based on vacuum properties, described by gluonic field correlators $38-52$ and the key role is played by correlators of colorelectric fields $D^{E}$ and colormagnetic fields $D^{H}$, which provide colorelectric confinement (CEC) with the string tension $\sigma^{E}(T)$ and colormagnetic confinement (CMC) with the string tension $\sigma^{H}(T)$. The latter being calculated from field correlators and on the lattice, grows with $T, \sigma^{H}(T) \sim g^{4}(T) T^{2}$ and insures the strong interaction at large $T$ mentioned above. It is interesting to note, that in the FCM the crossover phenomenon is connected with the gradual vanishing of the vacuum confining correlator $D^{E}(z)$ (and the resulting string tension $\sigma^{E}(T)$ ) with the growing temperature. The same phenomenon of the "melting confinement" can be observed in the $\mathrm{SU}(3)$ gluodynamics [49], where the string tension $\sigma(T)$, measured on the lattice [53 56] is also decreasing with $T$, but in the case of SU(3) it cannot smoothly match the fast growing gluon pressure (in contrast to the slowly growing glueball pressure due to large glueball masses $\gtrsim 2 \mathrm{GeV}$ ). As a result, one has a weak first order transition in $\mathrm{SU}(3)$ [49, while in the $n_{f}=2+1$ QCD with low mass mesons the smooth matching of pressure is achievable in the course of transition.

As a proof of this picture one can use the quark condensate vanishing with $T$ [19,23,57) in the same way, as $\sigma(T)$ (see e.g. Fig.4 in [53],Fig.6 in [55] with Fig.4 in [19]), which is connected with confinement via $\langle\bar{q} q\rangle \sim \sigma^{3 / 2}[5860]$, and almost the same reasoning can extend this connection to nonzero $T$.

One can see many important questions in QCD that could be investigated by the FCM, and we will focus on one of them: the main task of this paper is to give a self-consistent description of QGP at nonzero $\mu_{B}$.

We shall use below the thermodynamic formalism exploited before for the gluon plasma in 48 50 and extended to the QGP case in Refs. [51, 52].

The paper is organized as follows: In section 2 we introduce the FCM in the case of finite temperature and chemical potential. In section 3 we calculate the Polyakov line $(L(T))$ in the case of $(2+1) n_{f}$ QCD. In section 4 we extend the FCM formalism to nonzero $\mu_{B}$. In section 5 we compare our results with lattice data at zero and finite baryon chemical potential. Section 6 is devoted to conclusions and the outlook.

\section{The Field Correlator Method at Finite temperature}

The field correlator method(FCM) is very powerful tool for describing physics of QCD(see [61] for a recent review) which allows to formulate "confinement" or in other words to obtain the area law of the Wilson loop in terms of the vacuum background fields, with the field correlators $D^{E}, D^{H}$ ensuring colorelectric and colormagnetic confinement with the string tensions $\sigma^{E}$ and $\sigma^{H}$.As a result all hadron masses are defined in this method only by fermion masses and string tension $\sigma^{E}$.

All gluon fields $A_{\mu}$ in QCD in the framework of the background perturbation theory [62] can be divided into vacuum background part $B_{\mu}$ and perturbative part $a_{\mu}, A_{\mu}=B_{\mu}+a_{\mu}$, with $B_{\mu}$ contributing to $\sigma^{E}, \sigma^{H}$ while $a_{\mu}$ is treated in the background perturbation theory with the perturbative coupling constant $\alpha_{s}(Q)$, defined by the scale parameter $\Lambda_{Q C D}$.

For the hadron spectrum in QCD and for the QCD thermodynamics the basic role is played by the background fields $B_{\mu}$, while $a_{\mu}$ yield perturbative corrections. On the other hand in high momentum processes with $Q^{2} \gg M_{B}^{2}=2 \pi \sigma=O(1 \mathrm{GeV})$ the basic role is played by the perturbative fields $a_{\mu}$. The boundary $M_{B}^{2}$ found in [63] separates both types of dynamics and $\sigma$ itself defines the scale $\Lambda_{Q C D}[61]$. In this sense the fields $\overline{B_{\mu}}$ and $a_{\mu}$ can be associated with to the regions $Q^{2} \leq M_{B}^{2}$ and $Q^{2}>M_{B}^{2}$ respectively.

of quark matter studies.Possible discovery of quark stars will give much more opportunities for the QCD phase diagram studying 29,30 . 
In thermodynamics at temperatures $T \leq M_{B}$ the basic dynamics is given by the background fields $B_{\mu}$ which define both colormagnetic confinement(yielding CMC Debye screening) and Polyakov line interactions. In what follows we shall concentrate on these contributions, taking into account gluon exchange corrections. The fundamental role in FCM is played by the quadratic gluonic field correlator. It consists of two terms $D$ and $D_{1}$

$$
\begin{aligned}
D_{\mu \nu \lambda \rho}=g^{2} \operatorname{tr}_{a}<F_{\mu \nu}(x) \Phi(x, y) F_{\lambda \rho}(y) \Phi(y, x)>=\left(\delta_{\mu \lambda} \delta_{\nu \rho}-\delta_{\mu \rho} \delta_{\nu \lambda}\right) D(x-y) & +\frac{1}{2}\left[\frac{\partial}{\partial_{\mu}}\left(x_{\lambda} \delta_{\nu \rho}-x_{\rho} \delta_{\nu \lambda}\right)\right. \\
& +(\mu \lambda \leftrightarrow \nu \rho)] D_{1}(x-y) .
\end{aligned}
$$

Here the parallel transporter $\Phi(x, y)=P \exp \left(i g \int_{y}^{x} d u_{\nu} A_{\nu}(u)\right)$, and the fields $F_{i 4}, F_{4 i}$ refer to $D^{E}, D_{1}^{E}$ and $F_{i k}$ to the $D^{H}, D_{1}^{H}$ correlators. One can obtain string tension via $D^{E}, D^{H}$ :

$$
\sigma^{E, H}=\frac{1}{2} \int D^{E, H} d^{2} z
$$

At zero temperature $\sigma^{E}=\sigma^{H}$. Let's discuss in more detail the basic principles of FCM at finite temperatures. We must take into account that at finite temperatures the confinement-deconfinement transition occurs. In our formalism that means that electric string (or colorelectric correlator $D^{E}$ ) has to vanish. But there is no restrictions on the value of colormagnetic correlator (or alternatively on the existence of colormagnetic string tension $\sigma_{H}$ ). As shown by analytic [64] and lattice studies $\sigma_{H}$ grows quadratically with temperature. As a result for $T>T_{c}$ there is no confining string between colour charges, but there is still non-perturbative interaction between them i.e. colorelectric (CE) interaction, contained in the Polyakov line $\mathrm{L}(\mathrm{T})$, and the colormagnetic confinement $(\mathrm{CM})$ in a spatial projection of the Wilson loop. Analysis of physics of QGP in terms of FCM made in [44 47, 65, 66], also confirmed the important role of Polyakov loops for description of thermodynamic of GP and QGP. In [48 52$]$ also the CMC interaction was taken into account, providing a selfconsistent dynamical picture in a good agreement with lattice data. As for CMC it is the main interaction in QGP, operating above transition temperature, as was observed in lattice data [67], where the CMC correlators $\left\langle\operatorname{tr} F_{i k}(x) \Phi(x, y) F_{i k}(y)\right\rangle$ have been measured, see also [68] where $\sigma^{H}$ was studied on the lattice, and [64] where $\sigma^{H}$ was estimated in FCM.

It was found in [69] that CMC does not support white bound states in $q \bar{q}$ and $g g$ systems at zero temperature, however it can create the screening mass $M(T)$ of isolated quarks and gluons [48,51, 64, which grows with temperature so that the ratio $\frac{M(T)}{T}$ is constant up to the logarithmic terms.

As it was shown in $44-52$ the most convenient for description of QCD thermodynamics is the T-dependent path integral (worldline) formalism, where pressure can be written in the form 44, 47,49 (see Appendix 1 for details of derivation)

$$
P_{g l}=2\left(N_{c}^{2}-1\right) \int_{0}^{\infty} \frac{d s}{s} \sum_{n=1,2 . .} G^{n}(s) .
$$

Here s is the proper time, and for $G^{n}(s)$ one can obtain:

$$
G^{n}(s)=\int(D z)_{o n}^{\omega} \exp (-K) \hat{t r}_{a}<W_{\Sigma}^{a}\left(C_{n}\right)>
$$

where $K=\frac{1}{4} \int_{0}^{s} d \tau\left(\frac{d z^{\mu}}{d \tau}\right)^{2}$, and $W_{\Sigma}^{a}\left(C_{n}\right)$ is the adjoint Wilson loop defined for the gluon path $C_{n}$, which has both temporal (i4) and spacial projections (ij), and $\hat{t r}_{a}$ is the normalized adjoint trace. When $T>T_{c}$ the correlation function between $\mathrm{CE}$ and $\mathrm{CM}$ fields is rather week 44:

$$
\left\langle E_{i}(x) B_{k}(y) \Phi(x, y)\right\rangle \approx 0
$$

At this point when averaging $W_{\Sigma}^{a}\left(C_{n}\right)$ in (4) one should take into account that the paths of gluons at $n_{0} \neq 0$ are not closed, and there is a free piece of $n$ temporal steps, which should be connected by the gluon path to form a closed contour of Wilson loop, with the area law in the vacuum confining 
field. Therefore one can add before vacuum averaging a piece along time axis, which closes the gluon trajectory from $n_{0}$ to $n=0$ and back from $n_{q}=0$ to $n=n_{0}$ (which is an identical operation yielding a factor 1). In this way one obtains a product of a closed contour and a Polyakov line from 0 to $n_{0}$, and the vacuum averaging yields the expression for the factorized Wilson loops [49]:

$$
<W_{\Sigma}^{a}\left(C_{n}\right)>=L_{a d j}^{(n)}(T)<W_{3}>
$$

with $L_{a d j}^{(n)} \approx L_{a d j}^{n}$ for $T \leq 1 \mathrm{GeV}$. One can integrate out the $z_{4}$ part of the path integral $(D z)_{o n}^{\omega}=$ $\left(D z_{4}\right)_{o n}^{\omega} D^{3} z$, with the result

$$
G^{(n)}(s)=G_{4}^{(n)}(s) G_{3}(s), \quad G_{4}^{n}(s)=\int\left(D z_{4}\right)_{o n}^{\omega} e^{-K} L_{a d j}^{(n)}=\frac{1}{2 \sqrt{4 \pi s}} e^{-\frac{n^{2}}{4 T^{2} s}} L_{a d j}^{(n)}
$$

This factorization holds also for quarks and will be used below (with the change of the adjoint representation to the fundamental one).

The resulting gluon contribution is

$$
P_{g l}=\frac{2\left(N_{c}^{2}-1\right)}{\sqrt{4 \pi}} \int_{0}^{\infty} \frac{d s}{s^{3 / 2}} G_{3}(s) \sum_{n=1,2, \ldots} e^{-\frac{n^{2}}{4 T^{2} s}} L_{a d j}^{n}, G_{3}(s)=\int\left(D^{3} z\right)_{x x} e^{-K_{3 d}}<\hat{t r}_{a} W_{3}^{a}>
$$

The direct appearance of $L_{a d j}^{(n)}(T)$ in the thermodynamic potential is an important feature of the present non-perturbative formalism based on FCM. It was derived before in [44], when the CMC was not taken into account, and the origin of $L^{(n)}$ was associated only with the correlator $D_{1}^{E}|65|$. As will be shown below the mechanism of the Polyakov loop is much more complicated, and we shall compute $L(T)$ in a different way.

At this point we are coming to the problem of the CMC and its contribution to the gluon dynamics.

As it is well known 66 the CMC generates the non-perturbative Debye mass $M_{D}(T)$, connected to the CM string tension $\sigma^{H}(T)$, which is proportional to $T^{2}$

$$
\sigma^{H}(T)=\text { const } g^{4}(T) T^{2}
$$

as it was found on the lattice [68] and non-perturbatively in the Appendix of [64]. The exact calculation of $G_{3}(s)$, which should give the explicit dependence on $M_{D}(T)$ is however difficult, and therefore one can use approximations explained in Appendix 2.

The inclusion of colour-magnetic interaction leads to the generation of a non-perturbative Debye mass $M_{D}$ for gluons and quarks. For gluons $M_{a d j} \sim \sqrt{\sigma^{H}(T)}$, one can take it into account by an approximate expression for 3d Green function [49], which is derived in Appendix 2.

$$
G_{3}(s)=\frac{1}{(4 \pi s)^{3 / 2}} \sqrt{\frac{\left(M_{a d j}^{2}\right) s}{\sinh \left(M_{a d j}^{2}\right) s}}
$$

It should be mentioned that the resulting gluon pressure Eq. (8) is in a good agreement with the lattice data $[49]$.

In the non-interacting case i.e. $\sigma^{H}=0$ and $L_{a d j}=1$ one obtains the ideal gas pressure:

$$
P_{g l}=P_{0}=\frac{\left(N_{c}^{2}-1\right)}{45} \pi^{2} T^{4}
$$

For quarks one can write the expression in the same form as in (8), but with the quark mass term $e^{-m_{q}^{2} s}$ :

$$
\begin{gathered}
P_{f}=\sum_{q=u, d, s} P_{q}, P_{q}=\frac{4 N_{c}}{\sqrt{4 \pi}} \int_{0}^{\infty} \frac{d s}{s^{3 / 2}} e^{-m_{q}^{2} s} S_{3}(s) \sum_{n=1,2, \ldots}(-)^{n+1} e^{-\frac{n^{2}}{4 T^{2} s}} L_{f}^{n} \\
S_{3}(s)=\frac{1}{(4 \pi s)^{3 / 2}} \sqrt{\frac{\left(M_{f}^{2}\right) s}{\sinh \left(M_{f}^{2}\right) s}}, M_{a d j}^{2}=\frac{9}{4} M_{f}^{2}, L_{f}^{n}=\left(L_{a d j}^{n}\right)^{4 / 9}
\end{gathered}
$$


And again in the case of massless non-interacting fermions one obtains:

$$
P_{f}=N_{c} N_{f} \frac{7 T^{4}}{180}
$$

The full pressure reads as:

$$
P_{t o t}=P_{f}+P_{g l}
$$

Integrating over proper time interval ds in (11) and replacing the square root term in $(12)$ by an approximate exponential term [50,52] one obtains

$$
P_{f}=\sum_{q=u, d, s} P_{q}, \frac{P_{q}(T, \mu)}{T^{4}}=\frac{2 N_{c}}{\pi^{2}} \sum_{n} \frac{(-)^{n+1}}{n^{2}} L^{n} K_{2}\left(\frac{\bar{M} n}{T}\right) \frac{\bar{M}^{2}}{T^{2}},
$$

where $\bar{M}=\sqrt{m_{f}^{2}+\frac{M^{2}(T)}{4}}, \quad M(T)=a \sqrt{\sigma_{s}(T)}, a \approx 2|51,66|$.

To include the effects of the baryon chemical potential we should do the substitution in (11):

$$
L_{f}^{n} \rightarrow L_{f}^{n} \cosh (\mu n / T)
$$

\section{Polyakov line calculations.}

As we saw the thermodynamics of QGP in FCM is defined by two main ingredients: the nonperturbative screening masses $\mathrm{M}(\mathrm{T})$ are calculated via $\sigma^{H}(T)$ and known both analytically and on the lattice 66, 68. This part is especially important at high $\mathrm{T}$ due to the growth of $\sigma^{H}(T)$. Another important ingredient is the Polyakov line $\mathrm{L}$ with the dynamics defined by the field correlators $D^{E}$ and $D_{1}^{E}[65]$. Polyakov line was introduced in $[44-47]$ as a main dynamical ingredient of $Q G P$ and it is associated with the correlator $D_{1}(x)$, which produces the interaction term $V_{1}(r, T)$, with a nonzero asymptotics $V_{1}^{E}(\infty, T)$ so that the Polyakov line was written as $L(T)=\exp \left(-\frac{V_{1}(\infty, T)}{2 T}\right)$.

However, more careful analysis done in [70], has revealed, that there are 3 sources of the Polyakov line in the non-perturbative correlators (1), two of them are due to the correlator $D^{E}(x)$ and one due to $D_{1}^{E}(x)$, which also generates the perturbative part of $L(T)$. We relegate the detailed analysis of these sources to the Appendix 3, and ref. [70] and here we only conclude, that the main part of the contribution of $D_{1}^{E}(x)\left(V_{1}^{E}(\infty, T)\right)$ is cancelled by that of the saturated part of $D^{E}(x)$ and the resulting contribution can be associated with the confining interaction of the static charge of Polyakov line with a picked-up antiquark, which create the heavy-light system with mass $M_{H L}(T)$, so that one can continue the previous definition of $L(T)$ as

$$
L(T)=\exp \left(-\frac{V_{1}(\infty, T)}{2 T}\right) \rightarrow \exp \left(-\frac{M_{H L}(T)}{T}\right) .
$$

One of the ways to calculate $\mathrm{L}$ is to evaluate it via the heavy-light mass $M_{H L}$ [71]. Here we are using as in 71 the mass $M_{H L}(T)$, which is T-dependent due to the temperature dependent string tension $\sigma^{E}(T)$,studied repeatedly on the lattice 53 55, with the relation $M_{H L}(T) \sim \sqrt{\sigma^{E}(T)}$. To find $\sigma^{E}(T)$ explicitly one can use a connection between $\sigma^{E}$ and the quark condensate $\langle\bar{q} q\rangle$ found in 60, 61, which can be associated with the T-dependent quark condensate, since in the FCM approach the latter is produced by the scalar confinement 5581]. Indeed, the lattice data on $\sigma(T)$ 53 55] and $\bar{q} q(T)$ 19, 23, 57 show a similar behaviour.

We take the CE string tension in the massless quark limit related to the chiral condensate [60] as $|\langle\bar{q} q(T)\rangle|=$ const $(\sigma(T))^{3 / 2}$. Introducing a dimensionless parameter $a(T)$ as $\sigma(T)=\sigma(0) a^{2}(T)$, one has

$$
|\langle\bar{q} q\rangle(T)|=|\langle\bar{q} q\rangle(0)| a^{3}(T)
$$


As a result one has $M_{H L}(T)=M_{H L}\left(T_{0}\right) \frac{a(T)}{a\left(T_{0}\right)}$ and $L(T)=\exp \left(-\frac{M_{H L}(T)}{T}\right)$. The numerical data are shown in Fig 1 . The error band in Fig.1 corresponds to the accuracy of $a(T)$ in the lattice data in 23 and the solid black line is our "ideal" FCM line $L_{F C M}(T)$ which on one hand is close to the error band, and on the other hand as will be seen below in the paper, yields a good agreement with lattice data.

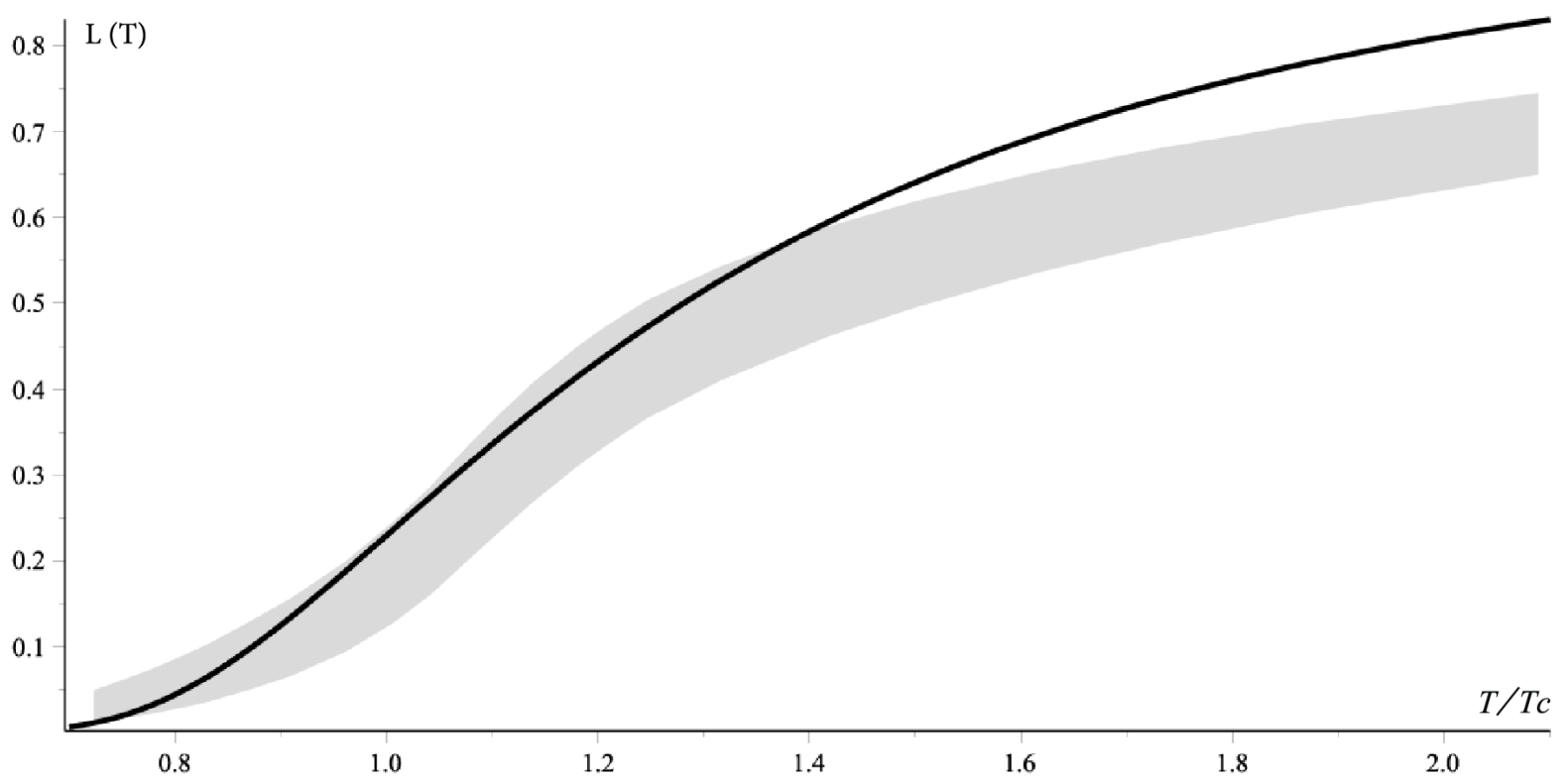

Figure 1: $\mathrm{L}(\mathrm{T})$ as a function of $T / T_{c}, T_{c}=160 \mathrm{MeV}$.Grey band corresponds to $L_{H L}(T)$ within the accuracy limits of a $(\mathrm{T})$. The solid black line is the "ideal" $L_{F C M}(T)$ used below in the paper.

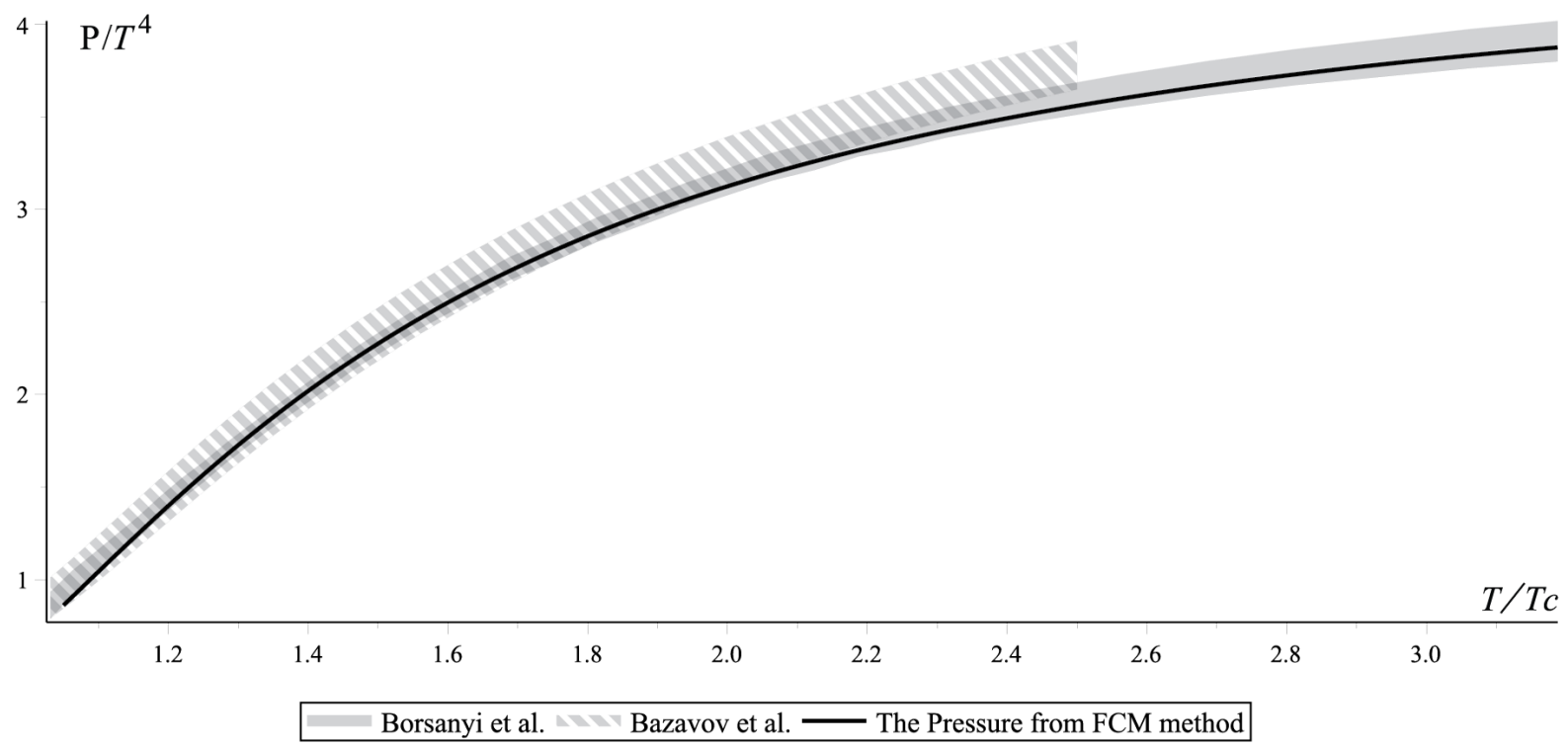

Figure 2: The ratio of the pressure to $T^{4}$ as a function of $T / T_{c}$. The grey band is the lattice data of Borsanyi et al. 20] and the striped band is the lattice data from Bazavov et al. [23]. 


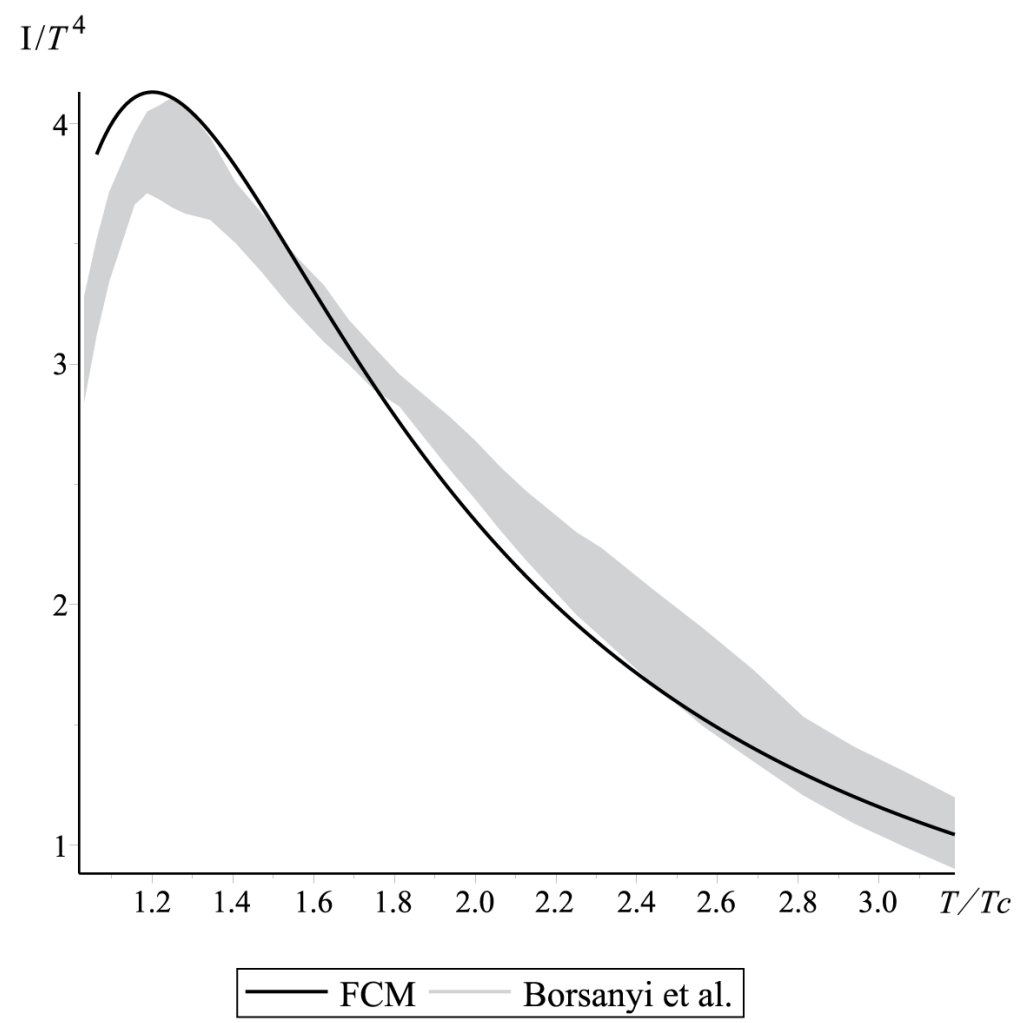

Figure 3: The ratio of the anomaly $(I=\epsilon-3 P)$ to $T^{4}$ in QGP as a function of $T / T_{c}$. The grey band is the lattice data of Borsanyi et al. [20].

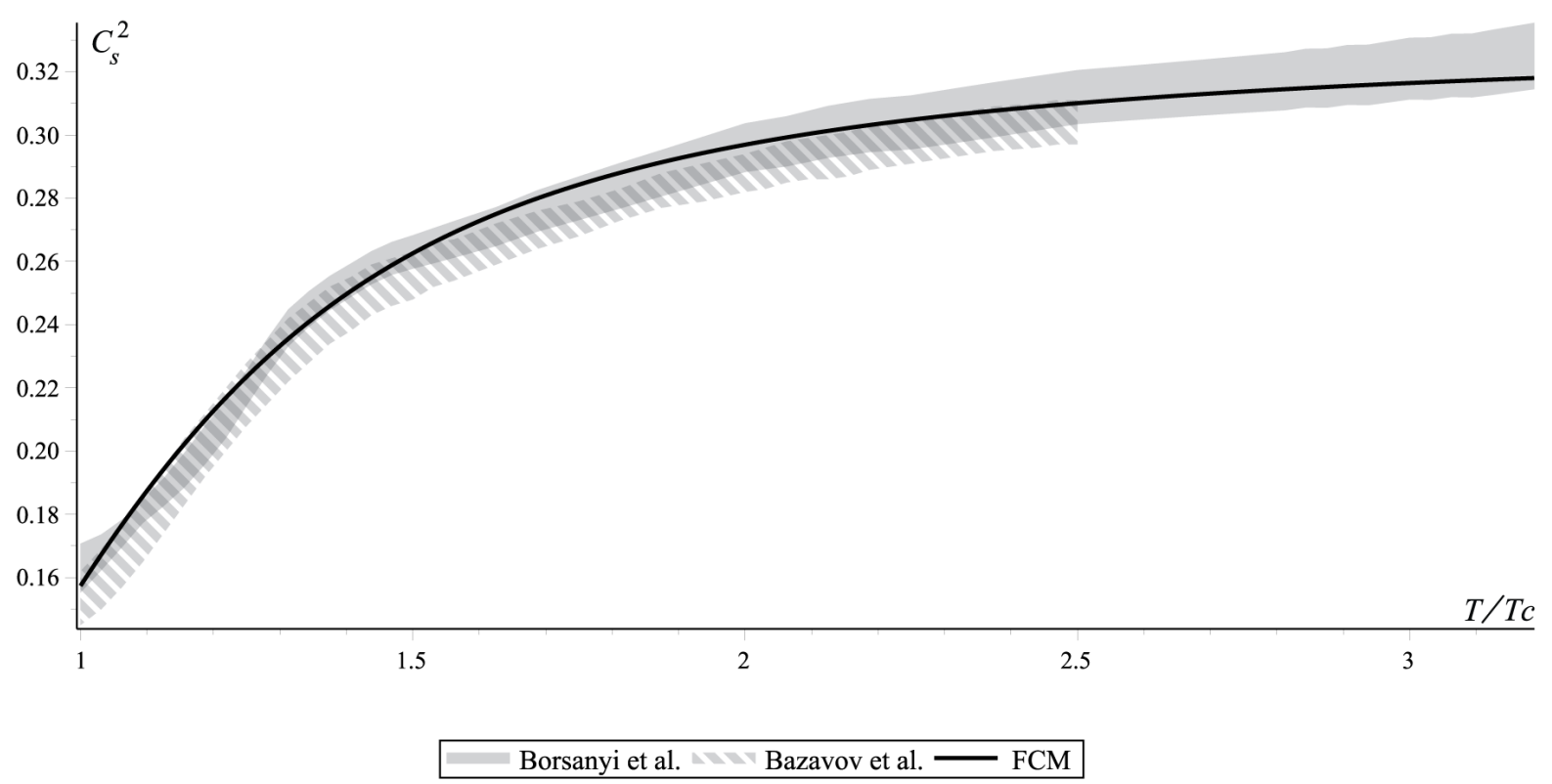

Figure 4: The square of the speed of sound in QGP as a function of $T / T_{c}$. The grey band is the lattice data of Borsanyi et al. [20] and the striped band is the lattice data from Bazavov et al. [23]. 


\section{The QCD thermodynamics at finite baryon chemical potential}

One of immediate tests of the FCM thermodynamics is the behaviour of the QGP pressure Fig 2 , the scale anomaly Fig 3 and the speed of sound Fig, 4 , where $L_{F C M}(T)$ and the CMC Debye mass is taken into account, in comparison with lattice data for zero and extension to non-zero baryon chemical potential. One can see that our predictions are in good agreement with lattice data at $\mu=0.3^{3}$

At this point we extend our results to a finite baryon chemical potential. We will use the definition of the baryon chemical potential in the same way as in [51] i.e. $\mu_{B}=3 \mu_{q}$ (we will not include a separate chemical potential for the strange quark). There is the possibility of comparison of our predictions with the Taylor expansion of $\mu[32$. We use the following assumption: according to [46,72] at small densities , $\mu_{B} \leq 300-400 \mathrm{MeV}$, we can neglect the influence of the baryon chemical potential on the Polyakov line.

The summation over $n$ in 15 can be done if one uses the integral representation

$$
K_{\nu}(z)=\frac{\left(\frac{z}{2}\right)^{\nu} \Gamma\left(\frac{1}{2}\right)}{\Gamma\left(\nu+\frac{1}{2}\right)} \int_{0}^{\infty} e^{-z \cosh t}(\sinh t)^{2 \nu} d t
$$

As a result one obtains as in [51]

$$
\frac{1}{T^{4}} P_{q}(T, \mu)=\frac{N_{c}}{\pi^{2}}\left(\xi_{1}^{(+)}+\xi_{1}^{(-)}\right)
$$

with

$$
\xi_{1}^{( \pm)}=\frac{1}{3}\left(\frac{\bar{M}}{T}\right)^{4} \int_{0}^{\infty} \frac{u^{4} d u}{\sqrt{1+u^{2}}\left[1+\exp \left(\frac{\bar{M}}{T} \sqrt{1+u^{2}}+\frac{V_{1}}{2 T} \pm \frac{\mu}{T}\right)\right]} .
$$

Changing the integration variable,

$$
\frac{\bar{M}}{T} \sqrt{1+u^{2}}=z+\frac{\bar{M}}{T}
$$

The expression 15 can be brought to the form:

$$
\begin{gathered}
\frac{P_{q}(T, \mu)}{T^{4}}=f_{+}(T, \mu)+f_{-}(T, \mu), \\
f_{ \pm}(T, \mu)=\frac{N_{c}}{3 \pi^{2}} \int_{0}^{\infty} \frac{d z\left(z^{2}+2 z \frac{\bar{M}}{T}\right)^{3 / 2}}{1+\exp \left(z+\frac{\bar{M}}{T}+\frac{V_{1}(T)}{2 T} \mp \frac{\mu}{T}\right)},
\end{gathered}
$$

where it is taken into account that $L=\exp \left(-V_{1}(T) / 2 T\right)$.

The expression (22) has no singularities at real $\mu$, but $f^{ \pm}$may get a singularity for imaginary chemical potentials for $\operatorname{Im}(\mu)=\pi T$ due to vanishing of the denominator in 23 at $z=-\frac{\bar{M}}{T}-\frac{V_{1}(T)}{2 T}$.

Hence one can conclude that in the normal situation with real $\mu$ and $L_{f}$ the singularity in $P(\mu, T)$ is absent, this conclusion implies that there is no critical point $T_{c}(\mu)$ in the domain of small baryon chemical potentials and the analytic structure is affected only by complex singularities. From this point of view, it seems that our consideration could be extended without any changes to large enough values of the chemical potential and temperatures $T \leq 1 \mathrm{GeV}$ if we take $\bar{M}$ and $L$ independent of $\mu$.

To test ourselves we have calculated the pressure at $\mu_{B}=100,200,300 \mathrm{MeV}$ and $\mu_{B}=400 \mathrm{MeV}$. As will be seen in the next section there is reasonable agreement between our predictions and lattice data, without significant changes in QGP state with growing $\mu_{B}$.

\footnotetext{
${ }^{3}$ We extended our results to rather high temperatures, just because we wanted to test our basic principles.

${ }^{4}$ The same is true for the speed of sound in gluodynamics 73 .
} 


\section{Results and discussion}

Below we show our results for the pressure and the sound velocity in comparison with the lattice data. As was discussed above, we obtained Polyakov line expression via connection with the heavy-light meson mass, derived from the quark condensate using eq. (17). The exploited form of the Polyakov line $L_{F C M}$ is shown in Fig. 1 together with the dark region $L_{H L}(T)$ derived from the quark condensate. One can see, that $L_{F C M}$ is close to the $L_{H L}$ within its accuracy region.

The data for $M_{f}(T)$ and $M_{a d j}(T)=\frac{3}{2} M_{f}(T)$ are taken from the exponential approximation of the square root expression in $\left[9,, 12\right.$, which was taken as $M_{f}(T)=1.6 \sqrt{\sigma^{H}}$, which is near the Debye mass value, obtained in $[66$ and ensures the high temperature behaviour of $P(T)$, which is impossible to reproduce without this $\mathrm{CMC}$ contribution.

One can see in Fig.2 the comparison of our FCM result for $P(T)$ with the lattice data [47, 50] for the zero baryon density. The resulting curves coincide within their accuracy limits.

Even more appealing is the agreement of our FCM results for the pressure for $\mu_{B}=0.1,0.2,0.3$ $\mathrm{GeV}$ in Fig 5 with the lattice data of [32] for the same values of $\mu_{B}$. One can conclude, that at low $\mu_{B}, \mu_{B} \leq 0.4 \mathrm{GeV}$ the FCM results predict a smooth behaviour of $P\left(T, \mu_{B}\right)$ without any hint of a singular point and this is in agreement with the analytic structure of $P\left(T, \mu_{B}\right)$ displayed in Eqs. (22, ) (23). At the same time these results agree with the similar conclusions of the lattice studies [32]. The slight disagreement with the lattice data $\mu_{B}=400 \mathrm{MeV}$ (black solid line) on Fig, 6 could be connected with renormalization of Polyakov line at finite baryon densities. For example on Fig 6 we also show the pressure(grey solid line) with Polyakov line, that is scaled similar to the [74].

One should notice that the lattice results for the pressure in 32 were obtained in the first order of the square of the chemical potential
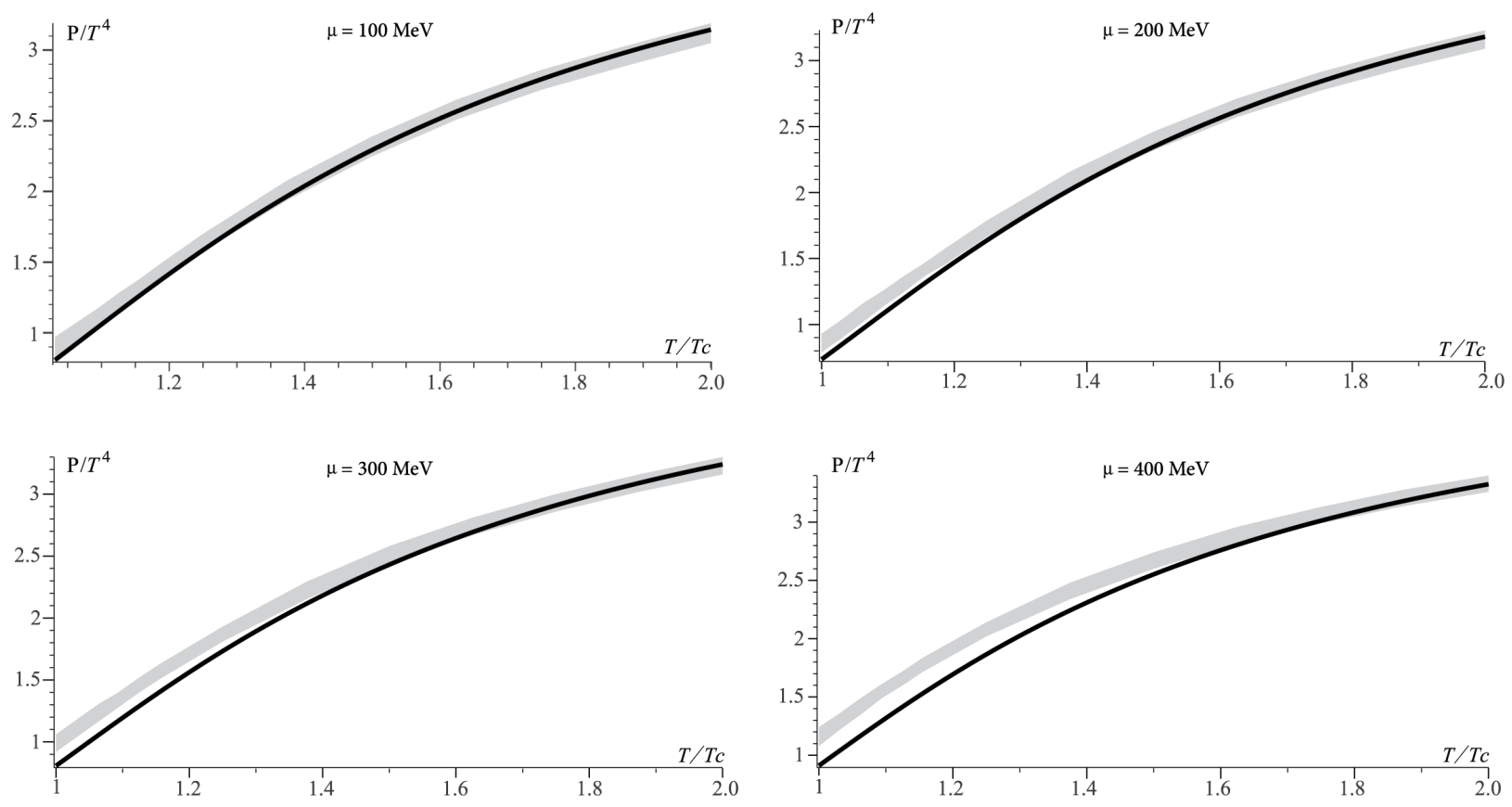

Borsanyi et al. - FCM

Figure 5: The ratio of QGP pressure to $T^{4}$ as a function of $T / T_{c}$ for $\mu_{B}=100,200,300,400 \mathrm{MeV}$. The grey bands are the lattice data of Borsanyi et al. from 32 at corresponding values of $\mu_{B}$

The square of the speed of sound, which for nonzero $\mu$ and in the isentropic condition can be 


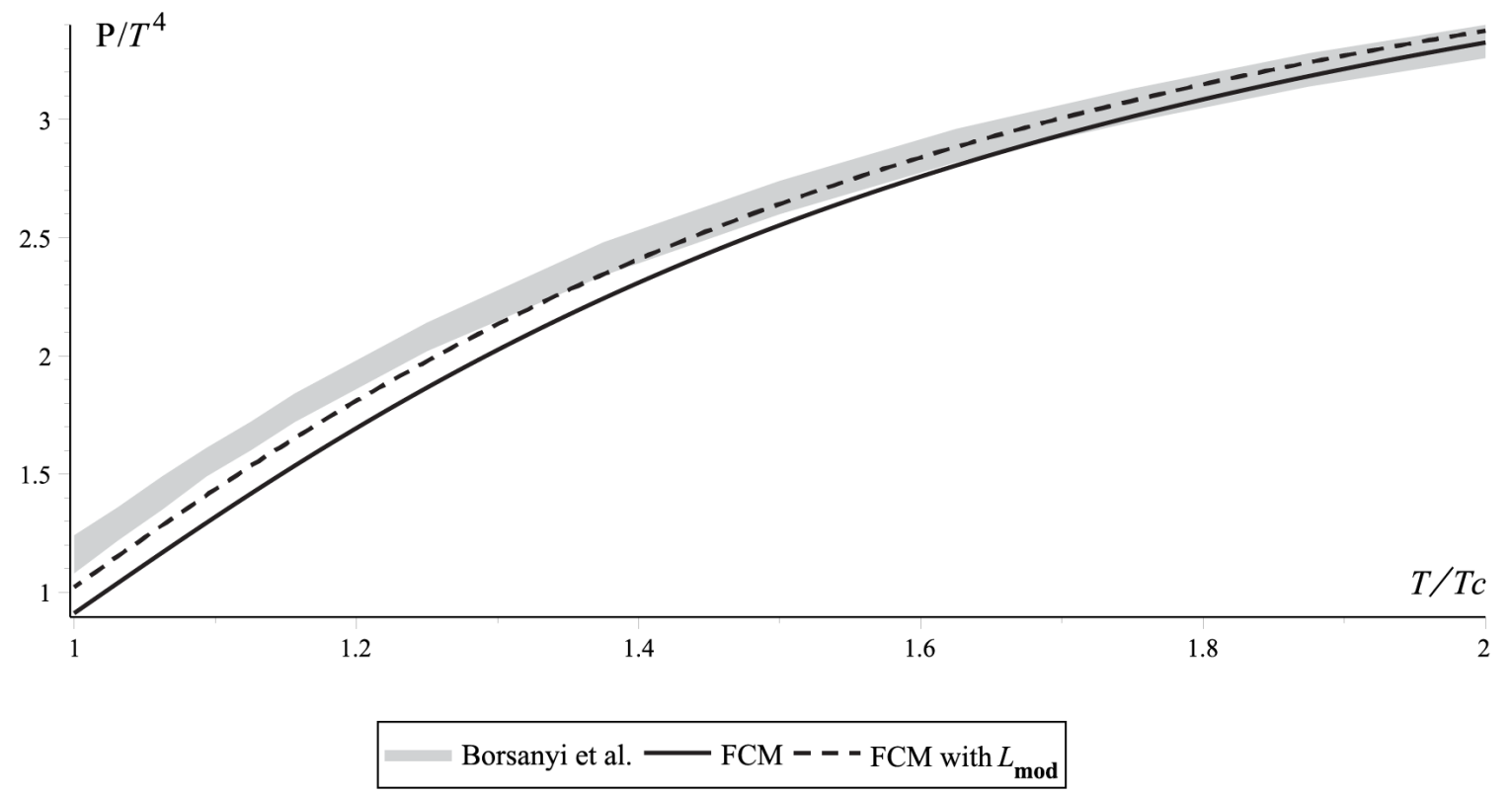

Figure 6: The ratio of QGP pressure to $T^{4}$ as a function of $T / T_{c}$ for $\mu_{B}=400 \mathrm{MeV}$ with $L_{F C M}$ (black line) and with Polyakov line that is scaled, similar to [74 (dashed line). The grey band is the lattice data of Borsanyi et al. from [32].

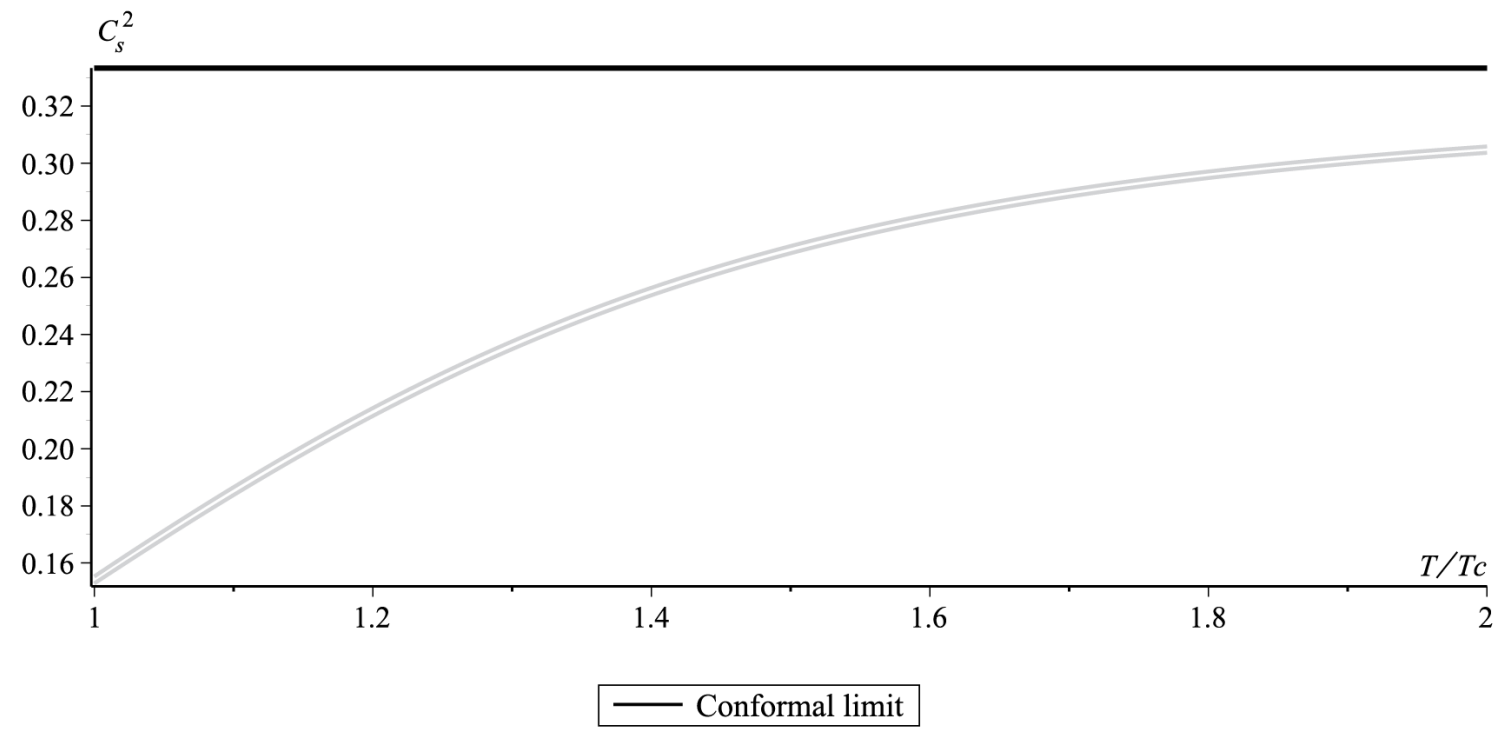

Figure 7: The width of solid line is the changing of the square of the speed of sound in the range $\mu_{B}=0 . .300 \mathrm{MeV}$

written as (see Appendix 4 for details of derivation).

$$
c_{s}^{2}=\frac{n^{2} \frac{\partial^{2} P}{\partial T^{2}}-2 s n \frac{\partial^{2} P}{\partial T \partial \mu}+s^{2} \frac{\partial^{2} P}{\partial \mu^{2}}}{(\varepsilon+p)\left(\frac{\partial^{2} P}{\partial T^{2}} \frac{\partial^{2} P}{\partial \mu^{2}}-\left(\frac{\partial^{2} P}{\partial T \partial \mu}\right)^{2}\right)},
$$


where we have defined:

$$
s=\frac{\partial P}{\partial T}, \quad n=\frac{\partial P}{\partial \mu}, \quad \varepsilon+P=T s+\mu n .
$$

We show in Fig 7 the speed of sound in the range $\mu_{B}=[0,300] \mathrm{MeV}$, where the width of the line is equal to the difference $c_{s}^{2}\left(\mu_{B}=300\right)-c_{s}^{2}\left(\mu_{B}=0\right)$. So from the FCM point of view the domain of low chemical potentials $\mu_{B}<400 \mathrm{MeV}$ is safe and could be described by Taylor expansion in baryon chemical potential $\mu_{B}$, because in this range this series converges, and the radius of convergences is defined by $\frac{\mu}{T}= \pm i \pi$ Roberge-Weiss point in Eq. (23).

\section{Conclusions and outlook}

The present paper is devoted to the effects of small baryon chemical potential $\mu_{B} \leq 400 \mathrm{Mev}$ in the dynamics of QGP.

It is an extension of the study of QCD thermodynamics at vanishing baryon density and is in the line of the series of papers 4452 where the QCD thermodynamics is worked out on the basis of FCM.

We have exploited above the FCM thermodynamics to calculate the QGP pressure at finite baryon density in the temperature range $1<T / T_{c}<2$, where $T_{c}=160 \mathrm{MeV}$.

Our basic dynamics was defined by two factors; the Polyakov line that is connected with $L_{H L}(T)=$ $\exp \left(-M_{H L} / T\right)$, and the colormagnetic confinement ( CMC) in the exponential form with the CMC quark mass $M_{D}=c \sqrt{\sigma^{H}(T)}$, where $c=1.6$ is close to the $q \bar{q}$ Debye mass in $|66|$ with $c=2$.

We have used for the heavy-light mass $M_{H L}(T)$ calculated from the $T$ - dependent string tension $\sigma^{E}(T)$, defined from the quark condensate $\langle q \bar{q}(T)\rangle$ measured on the lattice. The Polyakov line $L_{F C M}$ exploited in the paper is close to the accuracy limits $L=\exp \left(-M_{H L}(T) / T\right)$

We have demonstrated that the resulting pressure $P_{F C M}(T, \mu)$ is in good agreement with lattice data of the Budapest-Wuppertal [20,32] and Hot QCD groups [23] in both cases for zero and nonzero chemical potentials. We have also calculated changing in the speed of the sound that one could compare with FiG.[7] in [32.

From this point of view our analytic equations (8), 22, can be considered as an analytic counterpart of the corresponding lattice data.

All this implies the absence of a critical point in the studied range of $T$ and $\mu_{B}$ from the point of view of FCM method.

It should be noted however that we have used both $M_{D}$ and $M_{H L}$ independent of $\mu_{B}$ in the range $\mu_{B}<400 \mathrm{MeV}$.

The interesting region of high $\mu_{B}, \mu_{B}>1 \mathrm{GeV}$, is possibly hiding a completely different picture, with a singular behaviour of pressure and sound velocity, as it was found in [75]. However this phenomenon is strongly connected with a possible dependence of $L(\mu)$ and $M_{D}(\mu)$ recently studied on the lattice in $|72|$. These results are planned for the next paper.

\section{Acknowledgements}

We thank M. A. Andreichikov and B. O. Kerbikov, M. A. Zubkov,E.A.Fedina, R.A.Abramchuck and especially S.I.Blinnikov for very fruitful discussions.

This work was done in the frame of the scientific project, supported by the Russian Science Foundation Grant No. 16-12-10414. 


\section{Appendix 1.The FCM formalism in thermodynamics}

To start the FCM approach in the thermodynamics one can consider gluons and quarks in the background vacuum fields, which can contain both colorelectric and colormagnetic fields. Separating perturbative and vacuum gluonic fields, $A_{\mu}=B_{\mu}+a_{\mu}$, one can calculate the gluon and quark propagators in the loweest order in $a_{\mu}$ and take their vacuum average, as it was done in [76]. In this way one obtains the free energy and pressure in the lowest order of the standard perturbation theory, but with the full account of the averaged vacuum fields, given by correlators $D(x)$ and $D_{1}(x)$ in (1). Thus the free energy of gluons can be written via the gluon propagator in the form $[76$

$$
\begin{gathered}
\frac{1}{T} F_{0}(B)=\frac{1}{2} \ln \operatorname{det} G(B)-\ln \operatorname{det}\left(-D^{2}(B)\right)= \\
=\operatorname{tr}\left\{-\frac{1}{2} \int_{0}^{\infty} \zeta(s) \frac{d s}{s} e^{-s G(B)}+\int_{0}^{\infty} \zeta(s) \frac{d s}{s} e^{-s D^{2}(B)}\right\},
\end{gathered}
$$

where $G(B)$ is the gluon propagator and $D^{2}(B)$ ghost propagator in the background field

$$
\begin{gathered}
G_{\mu \nu}^{a b}=-D^{2}(B)_{a b} \delta_{\mu \nu}-2 g F_{\mu \nu}^{c}(B) f^{a c b}, \\
\left(D_{\lambda}\right)_{c a}=\partial_{\lambda} \delta_{c a}-g f_{b c a} B_{\lambda}^{b},
\end{gathered}
$$

while $\zeta(s)$ is the standard regularizing factor, $\zeta(s)=\left.\lim \frac{d}{d t} \frac{M^{2 t} s^{t}}{\Gamma(t)}\right|_{t=0}$, the exact form of it is inessential and it is not written in what follows.

Our final results require the vacuum field average of (A1.1) and the introduction of the temperature $T$. The vacuum averaging is to be done with quadratic combinations of fields $B_{\mu}$ in the exponent in A1.1, and to this end one can use the cluster expansion [77.

$$
\langle\exp f(B)\rangle_{B}=\exp \left\{\langle f(b)\rangle_{B}+\frac{1}{2}\left[\left\langle f^{2}(B)\right\rangle_{B}-\langle f(B)\rangle_{B}^{2}\right]+\ldots\right\}
$$

which is well converging, as shown in [41] due to the small vacuum correlation length $\lambda \lesssim 0.2 \mathrm{fm}$.

Next is the problem of the gluon (quark) Green's function in the external field, which can be represented as the path integral with the phase factor, containing the external field explicitly - this is called the Fock-Feynman-Schwinger representation (FFSR) [76], and has the following form in the simplest case of the ghost Green's function

$$
\left(-D^{2}\right)_{x y}=\left\langle x\left|\int_{0}^{\infty} d s e^{s D^{2}(B)}\right|_{y}\right\rangle=\int_{0}^{\infty} d s(D Z)_{x y} e^{-K} \hat{\Phi}(x, y) .
$$

Here

$$
K=\frac{1}{4} \int_{0}^{s} d \tau\left(\frac{d z_{\mu}}{d_{\tau}}\right)^{2}, \quad \hat{\Phi}(x, y)=\exp i g \int_{y}^{x} B_{\mu}(z) d z_{\mu}
$$

and the integral is taken along the trajectory of the ghost $y<z_{\mu}(\tau) \leq x$, and $(D z)_{x y}$ implies the path integral

$$
(D z)_{x y}=\prod_{m=1}^{N} \frac{d^{4} \zeta(m)}{(4 \pi \varepsilon)^{2}} \frac{d^{4} p}{(2 \pi)^{4}} e^{\left.i p\left(\sum_{m} \zeta(m)-x+y\right)\right)}
$$

where $\zeta(m)$ is the elementary piece of the path.

In a similar way the gluon propagator has the same representation $\mathrm{A} 1.5 \mathrm{~b}$ but with additional factor in A1.5 multiplying $\hat{\Phi}(x, y), \quad \hat{\Phi}(x, y) \rightarrow \hat{\Phi}(x, y) \exp \left(-2 i g \int_{0}^{s} d \tau \hat{F}_{B}(z(\tau))\right) \equiv \hat{\Phi}_{F}(x, y)$.

The next step is the introduction of the temperature $T$ within the Matsubara formalism. 
In the path integral the latter implies the only replacement: $(D z)_{x y} \rightarrow(D z)_{x y}^{w}$, where the upper index $w$ means the winding path integral, which comes from $x$ to the final point $y$ for the sequence of time intervals, $n \beta \equiv n / T$

$$
(D z)_{x y}^{w}=\prod_{M=1}^{N} \frac{d^{4} \Delta z(m)}{(4 \pi \varepsilon)^{2}} \sum_{n=0, \pm 1, \ldots} \frac{d^{4} p}{(2 \pi)^{4}} e^{i p\left(\sum_{m} \Delta z(m)-(x-y)-n \beta \delta_{\mu 4}\right)} .
$$

As it is seen in A1.8 the term with $n=0$ would yield the $T$-independent contribution to the pressure, contradicting the free gluon gas result, and should be omitted in what follows. The sum over $n= \pm 1, \pm 2$ gives the twice of the sum over $n=1,2,3, .$. As it is, only closed trajectories with $x=y$ are entering in the free energy (pressure).

As a result the pressure $P_{g l} V_{3}=-\left\langle F_{0}(B)\right\rangle$ can be written as follows:

$$
P_{g l}=T \int_{0}^{\infty} \frac{d s}{s} \frac{d^{4} x}{V_{3}}(D z)_{x x}^{w} e^{-K}\left[\frac{1}{2} \operatorname{tr}\left\langle\tilde{\Phi}_{F}(x, x)\right\rangle-\langle\operatorname{tr} \tilde{\Phi}(x, x)\rangle\right] .
$$

As it is clear in A1.9 the difference in the square brackets contains two effects: 1) the ghost reduction of the gluon d.o.f. 2) the gluon spin interaction corrections, since the operator $\hat{F}_{\mu \nu}$ entering in $\hat{\Phi}_{F}$ has the following representation

$$
-2 i \hat{F}_{\mu \nu}=2\left(\mathbf{S B}^{(1)}+\mathbf{S}^{(1)} \boldsymbol{\varepsilon}^{(1)}\right)_{\mu \nu},
$$

where $\mathbf{S}^{(1)}$ is the gluon spin operator and $\mathbf{B}^{(1)}, \boldsymbol{\varepsilon}^{(1)}$ are the background colormagnetic and colorelectric fields.

Therefore, neglecting at the first step the spin-dependent contribution one can replace the term in the square brackets simply by the adjoint Wilson loop, and as a result one obtains the representation, given below in A1.1). It is easy to understand the form A1.1), considering the free case with vacuum fields $B_{\mu} \equiv 0$. In this case, as demonstrated in the Appendix of [76], one obtains from the square brackets in $\mathrm{A} 1.9)\left(\frac{1}{2} \cdot 4-1\right)\left(N_{c}^{2}-1\right)=N_{c}^{2}-1$ and

$$
P_{g l}(B=0)=\varphi(B=0)=\left(N_{c}^{2}-1\right) \frac{T^{4} \pi^{2}}{45} .
$$

Summarizing, the final result for the gluon pressure can be written in the form Eq. (3) 44, 47, 49,

\section{Appendix 2 Colormagnetic confinement contribution to $S_{3}(s), G_{3}(s)$}

As one can see in (8), $G_{3}(s)\left(S_{3}(s)\right)$ contains the contribution of the adjoint (fundamental) loops respectively, which are subject to the area law, $\left\langle\hat{t r}_{i} W_{3}\right\rangle=\exp \left(-\sigma_{i} \operatorname{are} a(W)\right) i=$ fund, adj. Kinetic term is in $K_{3 d}$ in (8), so both $G_{3}(s)$ and $S_{3}(s)$ are proportional to the Green's functions of two color charges, connected by confining string, from one point $x$ on the loop to another (arbitrary) point, e.g. the point $u$ on the same loop. As it was shown in [51], one can represent $G_{3}(s)$ in the spectral sum form

$$
G_{3}(s)=\frac{1}{\sqrt{\pi s}} \sum_{\nu=0,1,2, . .} \psi_{\nu}^{2}(0) e^{-2 m_{\nu}^{2} s},
$$

where $m_{\nu}$ are eigenvalues of the Hamiltonian of two adjoint charges, connected by the string, and $\psi_{\nu}(x)$ is its eigenfunction in $2 \mathrm{~d}$.

As was discussed in [51, the spectral sum in A2.1 does not converge well, especially at large $T$, therefore one should calculate the combined effect of all terms. A simple example is given by the free case: $\sigma_{i}=0$. In this case one has 


$$
G_{3}^{(0)}(s)=S_{3}^{(0)}(s)=\frac{1}{\sqrt{\pi s}} \int \frac{d^{2} p}{(2 \pi)^{2}} e^{-2 p^{2} s}=\frac{1}{(4 \pi s)^{3 / 2}} .
$$

In this case one obtains the results for $P_{g l}, P_{q}$, which have been found before in 46

$$
P_{g l}^{(1)}=\frac{2\left(N_{c}^{2}-1\right) T^{3}}{\pi^{2}} \sum_{n=1,2, . .} \frac{L_{a d j}^{(n)}}{n^{4}}, \quad P_{q}^{(1)}=\frac{4 N_{c} T^{4}}{\pi^{2}} \sum_{n=1,2, . .}(-1)^{n+1} L_{f}^{(n)} \varphi_{q}^{(n)},
$$

where $\varphi_{q}^{(n)}$ is

$$
\varphi_{q}^{(n)}=\frac{n^{2} m_{q}^{2}}{2 T^{2}} K_{2}\left(\frac{n m_{q}}{T}\right)
$$

One can see in $\mathrm{A2.3}$ the Stefan-Boltzmann limit - for $L_{a d j}=L_{f}=1$.

There are two ways, how the CM confinement can be taken into account, suggested in [51]. Considering the oscillator interaction between the charges, one obtains

$$
G_{3}^{O S C}(s)=\frac{1}{(4 \pi)^{3 / 2} \sqrt{s}} \frac{M_{\mathrm{adj}}^{2}}{s h M_{\mathrm{adj}}^{2} s}
$$

and $S_{3}^{O S C}(s)$ is obtained from A2.5, replacing $M_{\text {adj }}$, by $M_{f}$. Here $M_{\text {adj }}=2 \sqrt{\sigma_{s}}=m_{D}(T)$, where $m_{D}(T)$ is the Debye mass, calculated in [66] in good agreement with lattice data.

A more realistic form obtains, when one replaces the linear interaction $\sigma_{s} r \rightarrow \frac{\sigma_{s}}{2}\left(\frac{r^{2}}{\gamma}+\gamma\right)$, varying the parameter $\gamma$ in the final expressions, imitating in this way linear interaction by an oscillator potential. Following [4] one obtains

$$
G_{3}^{\operatorname{lin}}(s)=\frac{1}{(4 \pi s)^{3 / 2}}\left(\frac{M_{\mathrm{adj}}^{2} s}{s h\left(M_{\mathrm{adj}}^{2} s\right)}\right)^{1 / 2}, \quad S_{3}^{\operatorname{lin}}(s)=\left.G_{3}^{\operatorname{lin}}(s)\right|_{M_{\mathrm{adj}} \rightarrow M_{f}} .
$$

Finally, substituting these expressions in (8), 11, , one obtains the equations for $P_{g l}^{\text {lin }}, P_{q}^{\text {lin }}$, containing the effects of CM confinement, which will be used in what follows. However to simplify the square-root expressions one can use for $M^{2} s \lesssim 1$ the approximation with the square root term replaced by the exponential, $\left(\frac{M^{2} s}{s h M^{2} s}\right)^{1 / 2} \approx \exp \left(-\frac{M^{2} s}{4}\right)$, which has a reasonable accuracy for $T<1 \mathrm{GeV}$.

\section{Appendix 3.Nonperturbative contribution to the Polyakov line}

The contribution of $D_{1}^{E}(x)$ and $D^{E}(x)$ to the $q \bar{q}(g g)$ interaction can be written in terms of local potentials $V_{D}(r), V_{D}^{\text {sat }}(r), V_{1}^{E}(r), V_{c}(r)$ and $V_{s} s(r)$, which will be neglected below. Here $V_{D}(r)=$ $\sigma r, \quad V_{c}(r)=-\frac{4 \alpha_{s}}{3 r}$, and

$$
\begin{aligned}
& V_{D}^{\text {conf }}(r, T)=2 r \int_{0}^{1 / T} d \nu(1-\nu T) \int_{0}^{r} d \xi D^{E}\left(\sqrt{\xi^{2}+\nu^{2}}\right) \\
& V_{D}^{\mathrm{sat}}(r, T)=2 \int_{0}^{1 / T} d \nu(1-\nu T) \int_{0}^{r} \xi d \xi D^{E}\left(\sqrt{\xi^{2}+\nu^{2}}\right) \\
& V_{1}^{\mathrm{sat}}(r, T)=\int_{0}^{1 / T} d \nu(1-\nu T) \int_{0}^{r} \xi d \xi D_{1}^{E}\left(\sqrt{\xi^{2}+\nu^{2}}\right)-V_{1}^{\mathrm{Coul}}(r, T) .
\end{aligned}
$$


The contributions of $V_{1}^{E}$ and $V_{D}^{\text {sat }}$ are strongly compensated as shown in [70], so that one is left with $V_{D}(r)$ and $V_{c}(r)$, the letter is effective mostly at large $T$, when $L$ is close to unity. Therefore one should take into account the potential $V_{D}(r)=\sigma r$, which gives rise to the heavy-light bound state with the mass $M_{H L}$ and $L=\exp \left(-\frac{M_{H L}}{T}\right)$. At this point it is important to fix the renormalization procedure of the contributing confinement and Coulomb interaction, which is similar in the lattice data of 78 and in our case and yields almost similar results for $L(T)$, as it is seen in 70 .

\section{Appendix 4 Derivation of eq.(24) for the sound velocity at finite $\mu$}

Below we are interested in the sound velocity $c_{s}$ at fixed isentropy $s / n_{B}, c_{s}^{2}=\left(\frac{\partial P}{\partial \varepsilon}\right)_{s / n_{B}} ; \frac{s}{n_{B}} \equiv \bar{s}$

$$
\left.\frac{\partial P}{\partial \varepsilon}\right|_{\bar{s}}=\frac{\frac{\partial P}{\partial T} d T+\frac{\partial P}{\partial \mu} d \mu}{\frac{\partial \varepsilon}{\partial T} d T+\frac{\partial \varepsilon}{\partial \mu} d \mu}, \quad \bar{s}=\frac{\frac{\partial P}{\partial T} / \mu}{N}=\text { const } .
$$

Taking into account the isentropic condition

$$
\begin{gathered}
d\left(\frac{s}{n_{B}}\right)=0=d\left(\frac{\frac{\partial P}{\partial T}}{\frac{\partial P}{\partial \mu}}\right)=\frac{d\left(\frac{\partial P}{\partial T}\right) \frac{\partial P}{\partial \mu}-\frac{\partial P}{\partial T} d \frac{\partial P}{\partial \mu}}{\left(\frac{\partial P}{\partial \mu}\right)^{2}}= \\
=\frac{\frac{\partial P}{\partial \mu}\left(\frac{\partial^{2} P}{\partial T^{2}} d T+\frac{\partial^{2} P}{\partial T \partial \mu} d \mu\right)-\frac{\partial P}{\partial T}\left(\frac{\partial^{2} P}{\partial \mu \partial T} d T+\frac{\partial^{2} P}{\partial \mu^{2}} d \mu\right)}{\left(\frac{\partial P}{\partial \mu}\right)^{2}}=0
\end{gathered}
$$

one obtains the relative change of $T$ and $\mu \div \frac{d T}{d \mu}$,

$$
\frac{d T}{d \mu}=\frac{\frac{\partial^{2} P}{\partial \mu^{2}} \frac{\partial P}{\partial T}-\frac{\partial^{2} P}{\partial \mu \partial T} \frac{\partial P}{\partial \mu}}{\frac{\partial^{2} P}{\partial T^{2}} \frac{\partial P}{\partial \mu}-\frac{\partial^{2} P}{\partial \mu \partial T} \frac{\partial P}{T}}=\frac{a}{b}
$$

As a result one obtains from (A4.1) (dividing numerator and denominator by $D \mu$ )

$$
c_{s}^{2}=\frac{\frac{\partial P}{\partial T} a+\frac{\partial P}{\partial \mu} b}{\frac{\partial \varepsilon}{\partial T} a+\frac{\partial \varepsilon}{\partial \mu} b},
$$

where $a, b$ are given in $\mathrm{A} 4.3$.

Now taking into account that $s=\frac{\partial P}{\partial T}, n=\frac{\partial P}{\partial \mu}, \quad \varepsilon+P=T s+\mu n$, one obtains the final form, given in the text.

$$
c_{s}^{2}=\frac{n^{2} \frac{\partial^{2} P}{\partial T}-2 s n \frac{\partial^{2} P}{\partial T \partial \mu}+s^{2} \frac{\partial^{2} p}{\partial \mu^{2}}}{(\varepsilon+P)\left(\frac{\partial^{2} P}{\partial T^{2}} \frac{\partial^{2} P}{\partial \mu^{2}}-\left(\frac{\partial^{2} P}{\partial T \partial \mu}\right)^{2}\right)}
$$

\section{References}

[1] STAR Collaboration: J. Adams, et al.Experimental and Theoretical Challenges in the Search for the Quark Gluon Plasma: The STAR Collaboration's Critical Assessment of the Evidence from RHIC Collisions, Nucl. Phys. A 757, 102 (2005), arXiv:nucl-ex/0501009. 
[2] PHENIX Collaboration, K. Adcox, et al.,Formation of dense partonic matter in relativistic nucleus-nucleus collisions at RHIC: Experimental evaluation by the PHENIX collaboration, Nucl.Phys. A 757, 184 (2005) arXiv:nucl-ex/0410003.

[3] I. Arsene et al., BRAHMS collaboration,Quark Gluon Plasma an Color Glass Condensate at RHIC? The perspective from the BRAHMS experiment. Nucl.Phys.A 757:1-27,(2005), arXiv:nuclex/0410020.

[4] M.Gyulassy, L.McLerran, Nucl. Phys. A 750, 30 (2005) arXiv:nucl-th/0405013.

[5] B.B.Back et al., (PHOBOS), Nucl. Phys. A 757, 28 (2005) arXiv:nucl-ex/0410022.

[6] E.V Shuryak, Rev. Mod. Phys. 89, 035001 (2017) arXiv:0807.3033v2.

[7] P. Braun-Munzinger, V. Koch, T. Schafer, and J. Stachel, Phys. Rept.621, 76 (2016), arXiv:1510.00442.

[8] Wit Busza, Krishna Rajagopal, Wilke van der Schee,MIT-CTP/4892, arXiv:1802.04801.

[9] R. Pasechnik, M. Šumbera, Universe 3, 7 (2017) arXiv:1611.01533.

[10] R. D. Pisarski and F. Wilczek, Phys. Rev. D 29, 33841 (1984).

[11] G. F. Chapline, M. H. Johnson, E. Teller, and M. S. Weiss, Phys. Rev. D 8, 4302 (1973).

[12] John C. Collins and M. J. Perry, Phys. Rev. Lett. 34, 1353 (1975).

[13] T. D. Lee, Phys. Rev. D 19, 1802, (1979).

[14] N. Cabibbo, G. Parisi, Phys. Lett. B 59, 67 (1975).

[15] E. V. Shuryak, Sov. Phys. JETP 47, 212 (1978), [Zh. Eksp. Teor. Fiz. 74, 408 (1978)].

[16] R.Snellings, New J.Phys.13, 055008 (2011) arXiv:1102.3010.

[17] Derek A. Teaney arXiv:0905.2433.

[18] T.Hirano, M. Gyulassy, Phys. A 769, 71-94 (2006) arXiv:nucl-th/0506049.

[19] S. Borsanyi, Z. Fodor, C.Hoelbling, S. D Katz, S. Krieg, C. Ratti,K.K. Szabo .,10.1007/JHEP 09, 073 (2010) arXiv:1005.3508.

[20] S.Borsanyi, Z.Fodor, C.Hoelbling,Phys. Lett. B 730, 99-104 (2014) arXiv:1309.5258 [hep-lat].

[21] F.Karsch, J. Phys. Conf. Ser. 46, 122-131 (2006); J.Goswanu, et al, Conference C18-07-22; arXiv:1811.02494.

[22] Owe Philipsen, Prog. Part. Nucl. Phys. 70, 55 (2013), arXiv:1207.5999,

[23] A.Bazavov,T.Bhattacharya, C. DeTar et al.,Phys. Rev.D 90, 094503 (2014),arXiv:1407.6387.

[24] S. Borsanyi, G.Endrodi, Z.Fodor, A.Jakovac, S. D. Katz, S.Krieg, C.Ratti, K.K. Szabo, JHEP 1011, 077,(2010), arXiv:1007.2580.

[25] Y. Aoki, G. Endrodi, Z. Fodor, S.D. Katz, K.K. Szabo, Nature, 443, 675 (2006), arXiv: heplat/0611014.

[26] P.Parotto, M.Bluhm, D.Mroczek et al, MIT-CTP-5015, arXiv:1805.05249.

[27] Tobias Fischer, Niels-Uwe F. Bastian, Meng-Ru Wu, Petr Baklanov, Elena Sorokina, Sergei Blinnikov, Stefan Typel, Thomas Klähn, David B. Blaschke,https://www.nature.com/articles/s41550018-0583-0 
[28] LIGO Scientific and Virgo Collaborations, Phys.Rev.Lett. 119 (2017) no.16, 161101, arXiv:1710.05832 [gr-qc].

[29] N. Itoh, Prog. Theor. Phys., 44, 291-292 (1970)

[30] E.Witten,Phys. Rev. D 30, 272 (1984).

[31] A. Bazavov, H.-T. Ding, P. Hegde et al., Phys. Rev. D 95, 054504 (2017) arXiv:1701.04325.

[32] Sz. Borsanyi, G. Endrodi, Z. Fodor, S. D. Katz, S. Krieg, C. Ratti, K. K. Szabo, JHEP 1208, 053 (2012), arXiv:1204.6710.

[33] J. Gunther, R. Bellwied, S. Borsanyi, Z. Fodor, S. D. Katz, A. Pasztor and C. Ratti, Nucl.Phys. A 967, 720 (2017) arXiv:1607.02493.

[34] V. G. Bornyakov, V. V. Braguta, E.-M. Ilgenfritz, A. Yu. Kotov, A. V. Molochkov, A. A. Nikolaev, JHEP 1803161 (2018), arXiv:1711.01869.

[35] V. V. Braguta, E.-M. Ilgenfritz, A. Yu. Kotov, A. V. Molochkov, A. A. Nikolaev, Phys. Rev. D 94114510 (2016), arXiv:1605.04090.

[36] V.V. Braguta, E.-M. Ilgenfritz, A.Yu. Kotov, B. Petersson, S.A. Skinderev, Phys. Rev. D 93, 034509 (2016), arXiv:1512.05873.

[37] V. V. Braguta, V. A. Goy, E.-M. Ilgenfritz, A. Yu. Kotov, A. V. Molochkov, M. Muller-Preussker, B. Petersson, JHEP 1506, 094 (2015) arXiv:1503.06670.

[38] H.G. Dosch, Phys. Lett. B 190, 177 (1987).

[39] H.G. Dosch, Yu.A. Simonov, Phys. Lett. B 205, 339 (1988).

[40] Yu.A. Simonov, Nucl. Phys. B 307, 512 (1988).

[41] A.Di Giacomo, H.G. Dosch, V.I. Shevchenko and Yu.A. Simonov, Phys. Rep. 372, 319 (2002),arXiv:0007223.

[42] Yu.A. Simonov, Phys. Usp. 39, 313 (1996) arXiv:hep-ph/9709344.

[43] D.S. Kuzmenko, V.I. Shevchenko, Yu.A. Simonov, Phys. Usp. 174, 3 (2004),arXiv:0310190.

[44] Yu.A.Simonov, Ann. Phys. 323, 783 (2008), hep-ph/0702266.

[45] E.V.Komarov, Yu.A. Simonov, Ann. Phys. 323, 1230 (2008), arXiv:hep-ph/0707.0781.

[46] Yu.A. Simonov, M.A.Trusov, Phys. Lett. B 650, 36 (2007), arXiv:hep-ph/0703277.

[47] A.V. Nefediev, Yu.A. Simonov, M.A.Trusov, Int. J. Mod. Phys. E 18, 549 (2009), arXiv:hep$\mathrm{ph} / 0902.0125$.

[48] N.O.Agasian, M.S.Lukashov and Yu.A.Simonov, Mod. Phys. Lett. A 31, 1050222 (2016); arXiv: 1610.01472 .

[49] N.O.Agasian, M.S.Lukashov and Yu.A.Simonov, Eur. Phys. J. A 53, 138 (2017); arXiv: 1701.07959.

[50] M.S.Lukashov and Yu.A.Simonov, JETP Lett. 105, 691 (2017); arXiv: 1703.06666.

[51] M.A. Andreichikov, M.S. Lukashov and Yu.A. Simonov, Int. J. Mod. Phys. A 33, 8 (2018), arXiv:1707.04631.

[52] M.A. Andreichikov, and Yu.A. Simonov, Eur. Phys. J. C 78, 5 (2018), arXiv:1712.02925. 
[53] O.Kaczmarek, F.Karsch, E.Laermann, M.Lutgemeier, Phys. Rev. D 62, 034021 (2000), heplat/9908010.

[54] P.Bicudo, and N.Caroso, Phys. Rev. D 85, 077501 (2012), arXiv: 1111.1317.

[55] P.Cea, L.Cosmai, F.Cuteri and A.Papa, JHEP, 6, 2 (2016); arXiv: 1511.01783.

[56] A. Bazavov, Y. Burnier and P. Petreczky, Nucl. Phys. A932, 117 (2014), arXiv:1404.4267.

[57] S.Borsanyi, C.Hoelbling, Z.Fodor, et al., POS Lattice 2010, 185 (2014), arXiv:1011.4230.

[58] Yu.A.Simonov, Phys. Atom. Nucl. 60, 2069 (1997).

[59] Yu.A.Simonov, Phys. At. Nucl. 67, 846 (2004); hep-ph/0302090.

[60] Yu.A.Simonov, Phys. At. Nucl. 67, 1027 (2004); hep-ph/0305281.

[61] Yu.A.Simonov, Phys. Rev. D 99, 056012 (2019); arXiv:1804.08946.

[62] B.S.De Witt, Phys. ReV. 162, 1195 (1967);

G.'t Hooft, Nucl. Phys. B 62, 444 (1973).

[63] Yu.A.Simonov, Phys. At. Nucl. 74, 1223 (2011), arXiv:1011,5386.

[64] Yu.A.Simonov, Phys. Rev.D, 096002 (2017); arXiv:1605.07060

[65] Yu.A.Simonov, Phys. Lett. B 619, 293 (2005),arXiv:hep-ph/0502078.

[66] N.O.Agasian, Yu.A.Simonov, Phys. Lett. B 639, 82 (2006) arXiv:hep-ph/0604004.

[67] M.D'Elia, A.Di Giacomo and E.Meggiolaro, Phys. Rev. D 67, 114504 (2003), hep-lat/0205018.

[68] G. Boyd, J. Engels, F. Karsch, E. Laermann, C. Legeland, M. Lutgemeier, B. Petersson, Nucl. Phys. B 469, 419 (1996), G. S. Bali, J. Fingberg, U. M. Heller, F. Karsch, and K. Schilling, Phys. Rev. Lett. 71, 3059 (1993), G.Boyd,J.EngelsF.Karsch,E.Laermann,C.Legeland,M.Lütgemeier,B.Petersson, Phys. Lett. B 346, 94 (1995).

[69] A.V.Nefediev and Yu.A.Simonov, Phys. Atom. Nucl. 71, 171 (2008).

[70] R.A.Abramchuk, Z.V.Khaidukov and Yu.A.Simonov, arXiv:1812.01998.

[71] Yu.S.Kalashnikova, A.V.Nefediev , Yu.A.Simonov, Phys.Rev. D 64 (2001) 014037, arXiv:hep$\mathrm{ph} / 0103274$

[72] M.D.'Elia, F.Negro, A.Rucci and F.Sanfilippo, arXiv:1907.09461.

[73] Z.V. Khaidukov, M.S.Lukashov, and Yu.A. Simonov,Phys. Rev. D 98, 074031(2018), arXiv:1806.09407.

[74] Massimo D'Elia, Francesco Di Renzo, Maria Paola Lombardo, Phys. Rev. D 76,114509,(2007) arXiv:0705.3814 [hep-lat]

[75] Z.V. Khaidukov and Yu.A. Simonov, arXiv:1811.08970,(2018).

[76] Yu. A.Simonov, Phys. At. Nucl. 58, 309 (1995), hep-ph/9311216.

[77] N.G.Van Kampen, Phys. Rep. C 24, 171 (1976).

[78] A. Bazavov, N. Brambilla, H.-T. Ding,H.P Schadler,A.Vairo and J.H.Weber Phys. Rev. D 93, 114502 (2016). 